\title{
EL CONTRATO DE ACCESO AL SISTEMA DE CIENCIA, TECNOLOGÍA E INNOVACIÓN: LA EQUIVALENCIA RETRIBUTIVA COMO DERECHO BÁSICO DEL INVESTIGADOR DOCTOR CONTRATADO A PROPÓSITO DE LA STSJ DEL PAÍS VASCO DE 22 DE OCTUBRE DE $2019^{1}$
}

\author{
Josep Moreno Gené2 \\ Profesor Titular de Derecho del Trabajo y de la Seguridad Social \\ Universitat de Lleida
}

\begin{abstract}
La STSJ del País Vasco de 22 de octubre de 2019 objeto de este comentario aborda la compleja cuestión de determinar quién es en el concreto supuesto enjuiciado el investigador comparable al investigador contratado mediante el contrato de acceso al sistema español de ciencia, tecnología e innovación -investigador "Ramón y Cajal"-, a los efectos de fijar su retribución.
\end{abstract}

The STSJ of the Basque Country of 22 October 2019 to which this comment refers, addresses the complex question of determining who is the researcher comparable to the researcher hired by means of the contract for access to the Spanish system of science, technology and innovation - "Ramón y Cajal"-, for the purposes of setting their remuneration.

IUSLabor 1/2021, ISSN 1699-2938, p. 143-163

DOI. 10.31009/IUSLabor.2021.101.06

Fecha envío: 29.11.2020 | Fecha aceptación: 25.01.2021

Title: The contract for access to the system of science, technology and innovation: remuneration equivalence as a basic right of the PhD researcher hired on the judgement of the High Court (STJS) of the Basque Country of 22 October 2019.

\footnotetext{
${ }^{1}$ Este trabajo se ha elaborado en el marco del proyecto de investigación RTI2018-097947-B-I00, concedido por el Ministerio de Ciencia, Innovación y Universidades que lleva por título "Nuevas tecnologías, cambios organizativos y trabajo: una visión multidisciplinar" (www.tecnologiaytrabajo.com).

${ }^{2} \mathrm{El}$ autor es miembro del grupo de investigación consolidado reconocido por la Generalitat de Cataluña "Social and Business Research Laboratory" (SBRLab). Ref. 2017 SGR 1572.
} 
Palabras clave: investigadores postdoctorales, contrato en prácticas, acceso al sistema español de ciencia, tecnología e innovación, igualdad retributiva

Keywords: postdoctoral researchers, internship contract, access to the Spanish system of science, technology and innovation, remuneration equality

\section{Sumario}

1. Los contornos del contrato de acceso al sistema de ciencia, tecnología e innovación y los derechos retributivos del contrato

2. La STSJ del País Vasco de 22 de octubre de 2019

2.1. El supuesto de hecho y la solución de instancia

2.2. La solución en suplicación: la aplicación de la equivalencia retributiva

3. La equivalencia retributiva como derecho básico del investigador contratado mediante el contrato de acceso al sistema español de ciencia, tecnología e innovación

4. Bibliografía 


\section{Los contornos del contrato de acceso al sistema de ciencia, tecnología e innovación y los derechos retributivos del contrato}

Una vez finalizada la primera etapa de la formación postdoctoral y ante las dificultades que tradicionalmente ha planteado la incorporación de estos investigadores postdoctorales al sistema español de ciencia, tecnología e innovación, el artículo 22 de la Ley de la Ciencia, la Tecnología y la Innovación (en adelante, Ley de la Ciencia) contempla una modalidad de contrato de trabajo específica para el personal investigador que recibe la denominación de "contrato de acceso al sistema español de ciencia, tecnología e innovación" (en adelante, contrato SECTI) y que, como su propio nombre indica, tiene por finalidad precisamente la de favorecer la incorporación de los investigadores postdoctorales al sistema español de ciencia, tecnología e innovación ${ }^{3}$.

Esta modalidad contractual se diseñó y más o menos se ajusta a los posibles beneficiarios de los diferentes programas de incorporación de doctores existentes, como por ejemplo, el Programa Ramón y Cajal, que exigen, entre otros requisitos, que los beneficiarios de las ayudas se encuentren en posesión del título de doctor, de modo que el contrato SECTI se limita a traducir al plano legislativo las necesidades contractuales que plantean los diferentes programas de incorporación de investigadores al sistema español de ciencia y tecnología. Pese a lo cual, muchas de estas convocatorias no se refieren específicamente a esta modalidad contractual para la contratación de los beneficiarios de las mismas, sino que se limitan a establecer de un modo genérico que las entidades contratarán laboralmente a los candidatos seleccionados "de acuerdo con la legislación laboral vigente y con la duración de los contratos y la retribución que, en su caso, se indiquen

\footnotetext{
${ }^{3}$ Vid. sobre esta modalidad contractual, entre otros, GONZÁLEZ DEL REY RODRÍGUEZ, Ignacio, "La contratación de investigadores por universidades públicas en la Ley de la Ciencia de 2011", Revista de Trabajo y Seguridad Social. CEF, núm. 404, 2016, LUJÁN ALCARAZ, José, "La contratación laboral del personal investigador ante la reforma laboral y la Ley de la Ciencia", en AAVV.: Impacto de la nueva legislación en la educación superior y la investigación, Universidad de Sevilla, Sevilla, 2012, MARINA JALVO, Belén, "Cuestiones esenciales del régimen jurídico del personal investigador al servicio de las universidades públicas tras la Ley 14/2011, de 1 de junio, de la Ciencia, la Tecnología y la Innovación", Revista General de Derecho Administrativo, núm. 30, 2012, MORENO GENÉ, Josep, "La contratación laboral de los investigadores en la Ley de la Ciencia, la Tecnología y la innovación: ¿el final del estado de excepción laboral en la ciencia?", Revista de Trabajo y Seguridad Social. CEF, núm. 340, 2011, SERRANO ARGÜELLO, Noemí, "Modalidades específicas de contratación laboral del personal investigador. De la precarización al desideratum de su estabilidad en el empleo y consolidación profesional", Revista General del Derecho del Trabajo y de la Seguridad Social, núm. 36, 2014 y SIRVENT HERNÁNDEZ, Nancy, "Las modalidades de contratación laboral previstas en la LCTI específicamente para el personal investigador", en BALLESTER LAGUNA, Fernando (Coord.), El estatuto profesional del personal investigador contratado en régimen laboral, Innap Investiga, Madrid, 2016.
} 
para cada actuación"4. Por el contrario, sí ha sido la modalidad contractual a la que han acudido muchas universidades para proceder a la incorporación de personal investigador doctor en el marco de sus planes propios de investigación ${ }^{5}$.

A diferencia del contrato predoctoral que supuso una auténtica novedad en nuestro ordenamiento jurídico introducida por la Ley de la Ciencia, el contrato SECTI encuentra un precedente inmediato en el contrato para la incorporación de investigadores al sistema de ciencia y tecnología regulado en el artículo 17.b) de la Ley 13/1986, de 14 de abril, de Fomento y Coordinación General de la Investigación Científica y Técnica (en adelante, Ley 13/1986), de conformidad con la redacción dada por la Ley 12/2001, de 9 de julio, de medidas urgentes de reforma del mercado de trabajo para el incremento del empleo y la mejora de su calidad (en adelante, Ley $12 / 2001)^{6}$, de modo que su entrada en vigor no supuso una modificación radical en este punto, sino una simple actualización o adaptación a las nuevas circunstancias de una modalidad contractual previamente existente.

El contrato SECTI se configura en la Ley de la Ciencia como una modalidad de contrato de trabajo específica del personal investigador que satisface una necesidad muy concreta de contratación consistente en que el personal investigador doctor contratado pueda desarrollar tareas de investigación que le permitan obtener un elevado nivel de perfeccionamiento y especialización profesional, consolidando de este modo su experiencia profesional. Pese a ello, como sucedía con el anterior contrato para la incorporación de investigadores al sistema de ciencia y tecnología, la regulación del contrato SECTI mantiene intensos vínculos con la regulación del contrato en prácticas prevista en el artículo 11 ET. En este punto, el artículo 22.1.f) de la Ley de la Ciencia prevé que "en lo no previsto en este artículo, será de aplicación el artículo 11.1 del Estatuto de los Trabajadores".

La remisión al contrato en prácticas que lleva a cabo la Ley de la Ciencia al regular el contrato SECTI permite plantear la cuestión de si esta modalidad contractual constituye

\footnotetext{
${ }^{4}$ Vid. artículo 14 de la Resolución de la Presidencia de la Agencia Estatal de Investigación por la que se aprueba la convocatoria de tramitación anticipada, correspondiente al año 2019, de diversas actuaciones contempladas en el Subprograma Estatal de Formación y en el Subprograma Estatal de Incorporación, del Programa Estatal de Promoción del Talento y su Empleabilidad, en el marco del Plan Estatal de Investigación Científica y Técnica y de Innovación 2017-2020 -Ayudas Ramón y Cajal, Ayudas Juan de la Cierva- Formación, Ayudas Juan de la Cierva-Incorporación y Ayudas para personal técnico de apoyo-. ${ }^{5}$ Vid. a título de ejemplo, la normativa de concursos para la contratación de profesorado de la Universidad Rovira y Virgili, adecuada, por acuerdo de 10 de julio de 2012, a la Ley 14/2011, de 1 de junio, de la Ciencia, la Tecnología y la Innovación, para incluir al personal de acceso al SECTI.

${ }^{6}$ Vid. LUJÁN ALCARAZ, José: “La contratación laboral del personal investigador...” cit. 204. Vid. sobre esta modalidad contractual, MORENO GENÉ, Josep, La actividad investigadora y la contratación laboral: una constante relación de desencuentros, Tirant lo Blanch, Valencia, 2005.
} 
una mera adaptación del contrato en prácticas común u ordinario a las características propias de la contratación de investigadores doctores o si, por el contrario, nos encontramos ante una modalidad contractual ciertamente independiente o desgajada del tronco común del contrato en prácticas previsto por el artículo 11.1 ET. Las extensas y relevantes particularidades previstas para el contrato SECTI respecto al contrato en prácticas ordinario o común parecen avalar esta segunda opción, configurándose al respecto una modalidad muy singular de contrato en prácticas dirigida a la contratación de los investigadores doctores ${ }^{7}$.

En este contexto, una de las materias que más separan al contrato SECTI del contrato en prácticas es el régimen retributivo. A tal efecto, la fijación para los trabajadores en prácticas de un salario inferior al del resto de los trabajadores ordinarios se ha perfilado como una nota común de los contratos formativos, incluido el de prácticas, en tanto que se ha entendido que los trabajadores contratados en prácticas se encuentran en un período laboral en el que contrastan la teoría con la práctica profesional, por lo que su productividad no es la misma, de modo que los beneficios que recibe el trabajador obtener una práctica profesional adecuada al nivel de estudios cursados- son compensados por el menor nivel de salario ${ }^{8}$. Esta finalidad justificaría, asimismo, que el salario previsto para el segundo año de vigencia del contrato en prácticas sea superior, puesto que el trabajador ya acumula una mayor práctica profesional que le hace más asimilable a un trabajador ordinario.

Por esta razón, llama especialmente la atención que el artículo 22.1.d) de la Ley de la Ciencia al regular la retribución del investigador doctor contratado a través de la modalidad contractual de acceso al sistema español de ciencia, tecnología e innovación establezca que "la retribución de este contrato no podrá ser inferior a la que corresponda al personal investigador que realice actividades análogas". Este precepto restablece, por tanto, el principio de equivalencia retributiva entre el personal investigador contratado a través de esta modalidad singular de contrato en prácticas dirigida a la contratación de los investigadores doctores y el personal investigador que realice idénticas o análogas

\footnotetext{
${ }^{7}$ En esta misma dirección, GONZÁLEZ DÍAZ, Francisco Antonio, "El personal investigador en formación..." cit. indica que "no se trata de un contrato en prácticas puesto que las particularidades diseñadas por el legislador lo alejan de la esencia de aquél, pero la aplicación subsidiaria del artículo 11.1 (...) nos permitiría hablar de un contrato en prácticas en cierta medida desvirtuado".

${ }^{8}$ Esta justificación ha permitido que el Tribunal Constitucional no haya entendido que estas desigualdades sean causa de discriminación, puesto que el Alto Tribunal no considera atentatorias al principio de igualdad aquellas regulaciones salariales desiguales que no impliquen ruptura del principio de equivalencia entre trabajo y salario, entendiendo que la distinción retributiva entre trabajadores en prácticas y los restantes trabajadores ordinarios operaría en este caso sobre dos modalidades contractuales que, por esta razón, ofrecen apreciables diferencias en la prestación de servicios respecto de la relación laboral común. Vid. por todas, STC 177/1993, de 31 de mayo.
} 
actividades mediante otra vía de vinculación laboral o funcionarial en la misma universidad u otro centro de investigación.

En definitiva, no se admite un diferente módulo y, por tanto, más bajo, para el cálculo del salario de este personal investigador doctor contratado, sino que se acude directamente al salario correspondiente al personal investigador que realice idénticas o análogas actividades en la misma universidad u otro centro de investigación. Tal decisión se debe, sin duda, a la consideración de que a través de esta modalidad contractual no se pretende incorporar a investigadores en fase de adiestramiento inicial, sino insertar en el sistema español de ciencia, tecnología e innovación a profesionales ya formados e incluso con cierta experiencia laboral.

En otros términos, el establecimiento de la equivalencia retributiva entre los investigadores contratados a través del contrato SECTI y el resto de investigadores de la entidad en la que se prestan los servicios permite constatar, de una manera definitiva y sin paliativos, que la finalidad fundamental de esta especie de contrato en prácticas no es la formativa, puesto que en este caso se contemplaría una retribución inferior. Por el contrario, pone de manifiesto que la formación de los investigadores doctores contratados mediante esta modalidad contractual ya es de suficiente nivel para que su trabajo sea considerado del mismo valor que el trabajo de otros investigadores, de modo que con esta modalidad contractual no se pretende complementar dicha formación, lo cual en todo caso es inherente a toda actividad investigadora, sino facilitar el acceso del investigador al mercado laboral y, en particular, al sistema español de ciencia, tecnología e innovación.

La retribución prevista para el contrato SECTI puede generar, sin embargo, importantes problemas de índole práctico. En primer lugar, resulta especialmente complicado identificar al investigador comparable; a tal efecto, se ha considerado que "la referencia legal es tan abierta que no puede equipararse con una clase/categoría de trabajador, ni parece que esta haya sido la intención del legislador, sino identificarlo con aquel que realice tareas análogas a las que en cada caso concreto sean realizadas por el

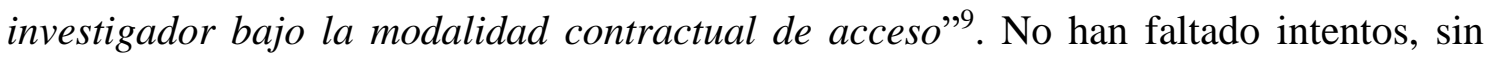
embargo, de asimilarlo a alguna figura concreta, en particular, en el ámbito de las universidades públicas a los profesores ayudantes doctores ${ }^{10}$.

Y, en segundo lugar, los diversos programas de ayudas a la investigación a través de los cuales se financian los contratos de los investigadores doctores, al fijar la cuantía de las ayudas con las que se financia la retribución de los mismos, se limitan a prever una

\footnotetext{
${ }^{9}$ Vid. SERRANO ARGÜELLO, Noemí, “Modalidades específicas de contratación...” cit. p. 110.

${ }^{10}$ En esta dirección, vid. LUJÁN ALCARAZ, José, "La contratación laboral del personal investigador..." cit. 206.
} 
cantidad fija, sin contemplar de ningún modo que dicha retribución sea equivalente a la retribución correspondiente a un investigador que realice actividades análogas, lo cual plantea la cuestión de qué sucede en aquellos supuestos en que la cantidad prevista en la correspondiente convocatoria de ayudas a la investigación con las que se financian los contratos SECTI es inferior a la retribución prevista para el investigador comparable ${ }^{11}$.

Precisamente, la STSJ del País Vasco de 22 de octubre de 2019 (núm. rec. 1699/2019) objeto de este comentario aborda ambas cuestiones, fijando quién es en el concreto supuesto enjuiciado el investigador comparable al investigador contratado mediante el contrato SECTI y a quien corresponde sufragar las diferencias salariales que pudieran existir entre la retribución prevista en el contrato SECTI, fijada a partir de la cuantía de las ayudas previstas en la convocatoria mediante la que se financia el contrato en cuestión y la retribución prevista para el investigador comparable.

\section{La STSJ del País Vasco de 22 de octubre de 2019}

\subsection{El supuesto de hecho y la solución de instancia}

La STSJ del País Vasco de 22 de octubre de 2019 (núm. rec. 1699/2019) conoce de un supuesto en que dos investigadores doctores se encuentran vinculados con la Universidad del País Vasco-Euskal Herriko Univertsitatatea (en adelante, UPV-EHU) bajo la modalidad de contrato SECTI (subprograma "Ramón y Cajal") con la categoría profesional de investigador Ramón y Cajal a tiempo completo, desde el 7 de noviembre de 2014 y desde el 26 de octubre de 2016, respectivamente. Tal y como se indica en el propio contrato de trabajo, el mismo se formaliza de acuerdo con lo previsto en el artículo 22 de la Ley de la Ciencia.

Dicho contrato se financia gracias a la ayuda obtenida por los investigadores en el marco de la Resolución de 1 de diciembre de 2014, de la Secretaría de Estado de Investigación, Desarrollo e Innovación, por la que se aprueba la convocatoria, correspondiente al año 2014, de diversas actuaciones contempladas en el Subprograma Estatal de Formación y en el Subprograma Estatal de Incorporación, del Programa Estatal de Promoción del Talento y su Empleabilidad, en el marco del Plan Estatal de Investigación Científica y Técnica y de Innovación 2013-2016. En concreto, los investigadores son beneficiarios de sendas ayudas obtenidas en el marco del programa "Ramón y Cajal".

El objeto del contrato es la prestación de servicios propios de la titulación de los referidos investigadores, consistentes en la realización de tareas de investigación orientadas a la

${ }^{11}$ Vid. MORENO GENÉ, Josep, “La contratación laboral...” cit. p. 106. 
obtención por el personal investigador de un elevado nivel de perfeccionamiento y especialización profesional que conduzcan a la consolidación de la experiencia profesional de los investigadores contratados.

En el contrato se prevé una evaluación consistente en el seguimiento por parte de la UPVEHU, que elaborará un informe relativo a los dos primeros años del contrato, incluyendo la descripción de lo realizado y el cumplimiento de los objetivos hasta la fecha del mismo con la conformidad del investigador contratado y el representante legal de la Universidad. De igual manera se procederá a los cuatro años del contrato. Estos informes serán evaluados por la Agencia Nacional de Evaluación y Prospectiva (en adelante, ANEP), siendo la evaluación determinante para mantener la continuidad en la financiación de los contratos. Con independencia de dicha evaluación, la Universidad podrá realizar un seguimiento científico-técnico anual de la actividad realizada y los objetivos cumplidos cuya superación será requisito necesario para el mantenimiento del contrato.

En el contrato formalizado con ambos investigadores se fija un salario de $31.600 €$ brutos anuales, distribuidos en 12 mensualidades, que se corresponde con la cuantía prevista en la convocatoria de ayudas a la investigación mediante la que se financia el contrato.

También de conformidad con la convocatoria de ayudas a la investigación mediante las que se financian los referidos contratos, los investigadores podrán prestar colaboraciones complementarias en tareas docentes en su misma institución o en otras por un máximo de 80 horas anuales, a petición propia y con la aprobación de su institución.

Ambos investigadores consideran que las retribuciones consignadas en el contrato de trabajo formalizado no se corresponden con las que efectivamente les correspondería percibir de conformidad con lo establecido por el artículo 22 de la Ley de la Ciencia, reclamando al respecto que les sean reconocidas las previstas para el personal doctor investigador contratado por la UPV-EHU, planteando para ello la correspondiente reclamación de cantidad por diferencias salariales. En otros términos, mantienen que la retribución que les corresponde no es la fijada en el contrato, establecida a partir de la correspondiente convocatoria de ayudas Ramón y Cajal $-31.600 €$ anuales- ${ }^{12}$, sino la que

\footnotetext{
12 Vid. artículo 20 de la Resolución de 1 de diciembre de 2014, de la Secretaría de Estado de Investigación, Desarrollo e Innovación, por la que se aprueba la convocatoria, correspondiente al año 2014, de diversas actuaciones contempladas en el Subprograma Estatal de Formación y en el Subprograma Estatal de Incorporación, del Programa Estatal de Promoción del Talento y su Empleabilidad, en el marco del Plan Estatal de Investigación Científica y Técnica y de Innovación 2013-2016. En dicho precepto se prevé que "La ayuda anual para cada uno de los contratos será de 33.720 euros. Esta ayuda se destinará necesariamente a cofinanciar el salario y la cuota empresarial de la Seguridad Social de los investigadores contratados durante cada una de las anualidades, consideradas independientemente. La retribución
} 
se desprenda del referido artículo 22 de la Ley de la Ciencia, que prevé en su apartado 1.d) que "la retribución de este contrato no podrá ser inferior a la que corresponda al personal investigador que realice actividades análogas".

Dicha pretensión es resuelta en instancia por la sentencia del Juzgado de lo Social núm. 1 de los de Vitoria que dictó sentencia el 9 de mayo de 2019 en la que se estimó la demanda interpuesta por los investigadores doctores, procediendo a homologar las retribuciones de estos investigadores a las previstas para los profesionales que prestan sus servicios como personal doctor investigador en la UPV-EHU, condenando a la universidad contratante a abonar las correspondientes diferencias salariales. A tal efecto, el magistrado de instancia se apoya en el artículo 22 de la Ley de la Ciencia, invocado por los investigadores que, como ya se ha indicado, señala que la retribución de los contratos SECTI no podrá ser inferior a la que corresponda al personal investigador que realice actividades análogas.

\subsection{La solución en suplicación: la aplicación de la equivalencia retributiva.}

Disconforme con la sentencia dictada en instancia, la UPV-EHU plantea el correspondiente recurso de suplicación que es resuelto por la STSJ del País Vasco de 22 de octubre de 2019 (núm. rec. 1699/2019) objeto de este comentario.

El recurso de la Universidad se apoya en la consideración de que no existe una homologación de los investigadores ahora demandantes con la figura de personal doctor e investigador contratado por la propia universidad. Para ello, se alegan diferencias entre ambas contrataciones que derivan de la propia normativa reguladora de cada una de las mismas, a saber, el artículo 22 de la Ley de la Ciencia, el artículo 27 de la Ley 3/2004, de 25 de febrero, del Sistema Universitario Vasco (en adelante, Ley 3/2004), el artículo 5 y 16 del Decreto 40/2008, de 4 de marzo, sobre régimen del personal docente e investigador de UPV/EHU (en adelante, Real Decreto 40/2008) y el artículo 17 de la Ley 13/1986.

Con la invocación de estos preceptos, la universidad pretende acreditar que "el personal contratado al amparo del artículo 22 de la Ley 14/2011, configura un tipo contractual que se homologa con aquéllos que siendo ya doctores, acceden al sistema de investigación, y lo hacen en una actividad de prácticas, como lo demuestra la remisión al artículo 11 ET que efectúa el precepto, de manera que no se pueden equiparar al personal que accede con otro sistema y realiza otras actividades de nivel más cualificado en el ámbito de la investigación".

mínima que deberán recibir los investigadores, que se deberá indicar en cada contrato, será de 31.600 euros brutos anuales". 
Sin embargo, a partir de la lectura de los preceptos indicados, el TSJ del País Vasco llega a la conclusión contraria, es decir, a la equiparación de ambas figuras de personal investigador en base a que se constata la realización de actividades análogas. A tal efecto, se indica que "la configuración de la investigación es y consiste en una actividad creativa que sistemáticamente sirve para incrementar el volumen de conocimientos en las diversas manifestaciones del mismo, y en tal sentido debiera demostrarse por la recurrente que existe una diferencia entre el aporte intelectual y creativo (...) que realizan los demandantes y el resto de investigadores con los que se les pretende "distanciar", pues ni consta que sean dependientes en su actividad de otros, ni que exista una diferencia en sus programas, proyectos o líneas de conocimiento". En definitiva, a criterio de la Sala de lo Social del TSJ del País Vasco, "no se aprecia una diferencia en la actividad que no sea, en su caso, la que proviene del tiempo de ejercicio de la profesión, elemento que no sirve para diferenciar una actividad de otra. Tampoco los resultados, los proyectos o los fines se muestran desiguales".

Por todo ello, en aplicación del artículo 22.1.d) de la Ley de la Ciencia, el TSJ del País Vasco condena a la Universidad a abonar las diferencias salariales existentes entre la retribución efectivamente percibida por los investigadores, fijada en sus respectivos contratos de trabajo en base a las correspondientes ayudas a la investigación mediante las que se financiaban dichos contratos, y la retribución prevista para la figura del personal doctor investigador en el Decreto 41/2008, por considerar que nos encontramos ante un personal investigador que realiza actividades análogas.

\section{La equivalencia retributiva como derecho básico del investigador contratado mediante el contrato de acceso al sistema español de ciencia y tecnología}

La diferenciación entre el contrato en prácticas ordinario o común previsto por el artículo 11.1 ET y el contrato SECTI introducido por la Ley de la Ciencia alcanza a la propia configuración de la finalidad perseguida por ambos contratos, puesto que mientras que puede afirmarse con mayor o menor rotundidad que el contrato en prácticas ordinario o común tiene como objetivo básico la formación del trabajador, al permitirle la obtención de una práctica profesional adecuada al nivel de estudios, el contrato SECTI, como su nombre indica, parece más bien una vía o un mecanismo de inserción del investigador en el mercado laboral y, por tanto, se configura como un instrumento de fomento del empleo 
de los investigadores ${ }^{13}$, más que de formación ${ }^{14}$. A tal efecto, aunque el contrato en prácticas ordinario o común también contempla una cierta finalidad de inserción laboral, se encuentra más dirigido a garantizar la formación del trabajador contratado.

En otros términos, el contrato SECTI pretende responder a las dificultades que existen para absorber el flujo de investigadores que produce el sistema, con lo que se convierte más en una vía para facilitar la carrera profesional de los investigadores al término de su formación, que en una vía de capacitación práctica o profesional. A tal efecto, se ha señalado que esta modalidad contractual constituye un "paso necesario (y no evitable para el investigador) para poder alcanzar, con posterioridad, la estabilidad en el empleo, ya sea mediante la contratación laboral fija o a través de una plaza de funcionario de carrera" $"$.

Esta función del contrato SECTI no resulta totalmente novedosa, puesto que la anterior modalidad contractual a través de la que se incorporaban los doctores al sistema de ciencia y tecnología, regulada en el artículo 17.1.b) de la Ley 13/1986, de conformidad con la redacción dada por la Ley 12/2001, ya se perfilaba como una modalidad contractual de inserción laboral, más que de formación ${ }^{16}$.

La primera manifestación del predominio de la finalidad de inserción laboral frente a la finalidad formativa del contrato SECTI se encuentra en la exigencia de que esta modalidad contractual sólo podrá concertarse "con quienes estén en posesión del título de doctor o equivalente" ${ }^{17}$ (artículo 22.1. a) de la Ley de la Ciencia). A tal efecto, la exigencia de una titulación del nivel del doctorado desvirtúa la dimensión formativopráctica de este contrato, ya que, habiéndose realizado los cursos de doctorado y el trabajo de investigación que conduce a la obtención del título de doctor, realmente cabe presumir que el sujeto posee la aptitud y la experiencia laboral suficientes para realizar trabajos de

\footnotetext{
${ }^{13}$ Vid. SERRANO ARGÜELLO, Noemí, "Modalidades específicas de contratación..." cit. p. 107. Vid. también, en relación con el anterior contrato de incorporación al sistema de ciencia y tecnología previsto en el artículo 17.1.b) de la Ley 13/1986, las STSJ de Canarias de 2 de septiembre de 2013 (núm. rec. 108/2013) y de Andalucía (Granada) de 18 de noviembre de 2015 (núm. rec. 1544/2015).

${ }^{14}$ Frente a ello, GONZÁLEZ DÍAZ, Francisco Antonio, "El personal investigador en formación..." cit. mantiene que "el investigador, contratado bajo esta modalidad, todavía se encuentra en un período formativo, si bien, con una mayor implicación investigadora y/o científica que en el contrato predoctoral en formación".

${ }^{15}$ Vid. SERRANO ARGÜELLO, Noemí, "Modalidades específicas de contratación...” cit. p. 107.

${ }^{16}$ Vid. sobre este paralelismo MORENO GENÉ, Josep, "El contrato de incorporación de investigadores al sistema de ciencia y tecnología", Revista de Derecho Social, núm. 29. 2005, p. 123 y ss.

${ }^{17}$ SERRANO ARGÜELLO, Noemí, "Modalidades específicas de contratación..." cit. p. 106, llama la atención sobre el hecho que a pesar de la mención que hace la norma al título "equivalente" al de doctor, no lo especifica si bien, cabe entender que, al menos, se refiere a homologaciones de titulaciones extranjeras asimilables al grado académico de doctor en España.
} 
investigación en los mismos términos que cualquier investigador que no esté contratado mediante esta especie de contrato en prácticas singular que es el contrato SECTI.

Es cierto, en todo caso, que tras la reforma del contrato en prácticas llevada a cabo por la Ley 35/2010, de 17 de septiembre, de medidas urgentes para la reforma del mercado de trabajo (en adelante, Ley 35/2010) se incorporó de forma expresa el título de doctor como titulación habilitante para la contratación en prácticas prevista en el artículo 11 ET. En consecuencia, un trabajador que se encuentre en posesión del título de doctor puede ser contratado indistintamente mediante el contrato en prácticas ordinario o común previsto en el artículo 11 ET o mediante el contrato SECTI introducido por la ley de la ciencia. No cabe duda que la finalidad que se persiga con esta contratación será la que determine en cada supuesto el recurso a una u otra modalidad contractual, de modo que la contratación de investigadores doctores deberá canalizarse preferentemente a través del contrato SECTI.

Junto con el requisito de titulación, el artículo 11 ET dispone que el contrato en prácticas sólo podrá concertarse "dentro de los cinco años, o siete años cuando el contrato se concierte con un trabajador minusválido, siguientes a la terminación de los correspondientes estudios". Este requisito de antigüedad de la titulación, sin embargo, ha sido expresamente excluido de la modalidad de contratación de investigadores prevista en el artículo 22 de la Ley de la Ciencia que establece que no serán de aplicación a esta modalidad contractual "los límites de cinco años, o de siete años cuando el contrato se concierte con un trabajador con discapacidad, a que se refiere el artículo 11.1 del Estatuto de los Trabajadores". En este punto se vuelve a poner en evidencia que la finalidad última del contrato SECTI no es tanto la formativa, sino fundamentalmente la de inserción en el mercado laboral, puesto que la supresión de este límite facilitará el acceso a esta modalidad contractual de aquellos investigadores que no hayan podido incorporarse al sistema español de ciencia, tecnología e innovación -mercado de trabajo de la ciencia- de forma inmediata a la obtención del título de doctor ${ }^{18}$.

No cabe duda que la supresión de este límite temporal, acompañada de la ausencia de una edad máxima para la formalización de este contrato y, fundamentalmente, de las peculiares características de la carrera investigadora, dará lugar con toda seguridad a la formalización de esta modalidad contractual con investigadores de avanzada edad y, fundamentalmente, con una amplia y acreditada experiencia teórico-práctica, lo que permite poner en tela de juicio una vez más el carácter formativo de esta modalidad contractual.

${ }^{18}$ Vid. GONZÁLEZ DÍAZ, Francisco Antonio, "El personal investigador en formación...” cit. 
Otra de las especialidades del contrato SECTI respecto al contrato en prácticas ordinario o común viene dada por el objeto de esta modalidad contractual. En este punto, el artículo 11.1 a) ET se limita a establecer que "el puesto de trabajo deberá permitir la obtención de la práctica profesional adecuada al nivel de estudios o de formación cursados", con lo que se pretende ofrecer al trabajador la posibilidad de completar la formación recibida con la experiencia propia del desempeño de un puesto de trabajo. Esta previsión genérica se concreta un poco más en el artículo 22.1.a) de la Ley de la Ciencia que prevé que el trabajo a desarrollar por el investigador contratado a través del contrato SECTI “consistirá primordialmente en la realización de tareas de investigación, orientadas a la obtención por el personal investigador de un elevado nivel de perfeccionamiento y especialización profesional, que conduzcan a la consolidación de su experiencia profesional"19.

Por tanto, la especialidad del contrato SECTI no viene dada tanto por la exigencia de que el trabajador contratado esté en posesión del título de doctor, con el cual, como ya se ha indicado anteriormente, también puede formalizarse un contrato en prácticas ordinario o común, sino por las funciones a desarrollar, es decir, las tareas de investigación, que deben permitir al investigador contratado alcanzar un alto nivel de perfeccionamiento y especialización profesional que conduzcan a la consolidación de su experiencia profesional. Este objeto del contrato pone de manifiesto, una vez más, que con esta modalidad contractual lo que fundamentalmente se persigue es la posterior inserción laboral del investigador en el sistema español de ciencia, tecnología e innovación.

A partir de estas premisas, algún sector doctrinal mantiene que la norma no lleva a cabo una delimitación de la prestación laboral, sino tan sólo de una parte, que parece la predominante, fundamental o principal, a saber, la realización de tareas de investigación. A tal efecto, se considera que la norma muestra una excesiva laxitud en la delimitación del objeto de esta modalidad contractual, el cual debería haberse precisado con mayor detalle. A partir de esta indefinición, se afirma que el objeto contractual cardinal de esta

\footnotetext{
${ }^{19}$ En este punto, llama la atención la laxitud de las funciones de estos investigadores que se admite en la STSJ de Galicia de 6 de febrero de 2015 (núm. rec. 4214/2014) cuando admite que "el desempeño ocasional en actividades diferentes de las que figuran en su contrato de trabajo, puede entrar dentro de las directrices y órdenes que el investigador principal pueda encomendar, para realizar actividades en otro grupo de investigación, que siempre van a estar enlazadas o conexionadas con el proyecto principal, es decir, que cualquiera de los trabajos realizados por el actor podían ser aprovechados tanto en la realización de su proyecto como en otros grupos de investigación, siempre que esas actividades sean meramente ocasionales, como así ha sido, sin que ello constituya fraude en la contratación, tal como invoca la parte recurrente. Y tampoco existe tal fraude por el hecho de que los proyectos de investigación del organismo demandado estén dentro de lo que se puede considerar su actividad normal y cotidiana, siempre que los mismos tengan autonomía y sustantividad propia, como ocurre en el presente caso, razón por la cual este motivo de recurso también debe ser desestimado".
} 
modalidad contractual "es un trámite hacia la consolidación investigadora (se esperará con aquella consolidación también la estabilidad en el empleo), pero no es el único, pero sin que el legislador nos indique cuáles son los otros objetos, los trabajos complementarios, al margen de la posibilidad de encargarle tareas docentes (como máximo de 80 horas anuales)" ${ }^{20}$. En este contexto, se ha considerado que las exigencias subjetivas y causales del contrato se compadecen mal con el adverbio "priomordialmente" con el que se pretende matizar la definición del objeto del contrato y por eso, "deberá ser interpretado de manera muy restrictiva, prácticamente limitada a la posibilidad de "colaboraciones complementarias en tareas docentes relacionadas con la actividad de investigación propuesta" 21.

A pesar de que el objeto de esta modalidad contractual consiste en la realización de tareas de investigación, como se acaba de anticipar, el artículo 22.1.e) de la Ley de la Ciencia también permite que el personal investigador que sea contratado a través del contrato SECTI pueda prestar colaboraciones docentes ${ }^{22}$. La posibilidad de colaborar en tareas docentes, sin embargo, únicamente se admite si se cumplen determinados condicionantes con los que se pretende asegurar que estas colaboraciones docentes no desvirtúen el objeto fundamental de esta modalidad contractual consistente en la realización de tareas de investigación, que permitan al investigador contratado alcanzar un alto nivel de perfeccionamiento y especialización profesional que conduzcan a la consolidación de su experiencia profesional. Bajo estas premisas, los requisitos exigidos para que los investigadores puedan prestar colaboraciones docentes son los siguientes: a) que se trate de colaboraciones complementarias a su actividad principal que es la investigación; b) que las colaboraciones docentes estén relacionadas con la actividad de investigación a desarrollar por el investigador contratado; c) que como máximo alcancen las 80 horas anuales; d) que exista acuerdo del departamento implicado y aprobación de la entidad para la que se prestan los servicios; y e) que se cumpla la normativa de incompatibilidades del personal al servicio de las administraciones públicas.

Como acertadamente se ha puesto de manifiesto, "las cautelas impuestas por el legislador, limitando las actividades docentes del investigador, controlando y autorizando la labor de enseñanza pretenden evitar, parece, la transformación impropia

\footnotetext{
${ }^{20}$ Vid. SERRANO ARGÜELLO, Noemí, “Modalidades específicas de contratación...” cit. p. 108.

${ }^{21}$ Vid. LUJÁN ALCARAZ, José, "La contratación laboral del personal investigador..." cit. p. 205.

${ }^{22}$ Como pone de manifiesto MOLINA HERMOSILLA, Olimpia, "Principales novedades en el ámbito de la gestión del personal docente e investigador. Un "pretendido modelo privilegiado" de acceso al empleo público", Revista de Trabajo y Seguridad Social. CEF, núm. 396, 2016, p. 58, “de esta posibilidad han venido beneficiándose ampliamente los investigadores contratados bajo esta modalidad por parte de las universidades, al objeto de poder acreditar como mérito a considerar la impartición de docencia, de cara a la obtención de las acreditaciones necesarias para acceder a plazas de profesorado universitario contratado o bien al Cuerpo de Funcionarios Docentes Universitarios".
} 
del investigador en "profesor" y erradicar posibles abusos en la encomienda de obligaciones docentes" 23 .

Una vez expuestas las características y, especialmente, el objeto del contrato SECTI ya estamos en disposición de valorar la decisión contenida en la STSJ del País Vasco de 22 de octubre de 2019 (núm. rec. 1699/2019) objeto de este comentario, consistente en homologar a los investigadores contratados mediante esta modalidad contractual con la figura del personal investigador doctor contratado previsto en la normativa universitaria vasca.

A tal efecto, si acudimos al artículo 27 de la Ley 3/2004 veremos que, entre otros aspectos, se prevé que: "el doctor investigador se dedicará fundamentalmente a la investigación", "para la contratación del personal investigador contratado se requerirá evaluación positiva previa" y que "el personal doctor investigador tendrá los mismos derechos que el profesor agregado". Asimismo, el artículo 16 del Real Decreto 40/2008 contiene las siguientes previsiones referidas a este colectivo de investigadores: "Para acceder a la condición de personal doctor investigador será preciso que las candidatas y candidatos acrediten 3 años de experiencia docente y/o investigadora y la evaluación positiva de la actividad docente e investigadora o prioritariamente investigadora, postdoctoral", "al personal investigador doctor se le podrá asignar obligaciones docentes hasta un máximo de 12 créditos anuales, siendo su objeto, principalmente, colaboraciones complementarias en tareas docentes relacionadas con la actividad de investigación propuesta, preferentemente de tercer ciclo" y que "el personal investigador doctor tendrá los mismos derechos que el profesorado agregado".

A partir de estas premisas, la universidad pretende diferenciar entre el personal contratado al amparo del artículo 22 de la Ley 14/2011 que define como aquéllos investigadores que, siendo ya doctores, acceden al sistema de investigación, y lo hacen en una actividad de prácticas, como lo demostraría la remisión al artículo 11 ET que efectúa el precepto, y el personal investigador doctor previsto en la normativa universitaria vasca que habría accedido a la universidad con otro sistema y realizaría otras actividades de nivel más cualificado en el ámbito de la investigación.

Este razonamiento de la universidad choca, sin embargo, con el hecho de que, como se ha puesto de manifiesto con anterioridad, a pesar de que el contrato SECTI se configura formalmente con un cierto carácter formativo, lo cierto es que las extensas y relevantes

${ }^{23}$ Vid. SERRANO ARGÜELLO, Noemí, "Modalidades específicas de contratación..." cit. p. 109. En la misma dirección, GONZÁLEZ DÍAZ, Francisco Antonio, "El personal investigador en formación..." cit. y SANMARTÍN RODRÍGUEZ, Javier, "El carácter laboral del profesor universitario", Thomson Reuters Aranzadi Civitas, Madrid, 2012, p. 72. 
particularidades previstas para esta modalidad contractual respecto al contrato en prácticas ordinario o común lo configuran como una modalidad muy singular de contrato en prácticas dirigida a la contratación de los investigadores doctores, en los que realmente, ya no predomina el aspecto formativo, sino el de inserción de los investigadores en el sistema español de ciencia, tecnología e innovación. A tal efecto, el elevado nivel de titulación exigida -título de doctor-, la no exigencia de que el contrato se formalice dentro de un arco temporal o que el objeto del contrato consista en la realización de tareas de investigación, orientadas a la obtención por el personal investigador de un elevado nivel de perfeccionamiento y especialización profesional, que conduzcan a la consolidación de su experiencia profesional, permiten constatar que no nos encontramos ante investigadores que se encuentren en una fase de formación en sus carreras investigadoras, sino con investigadores ya plenamente formados y que realizan su actividad en los mismos términos -idénticos, asimilables u homologables- que la pueden realizar otros investigadores que desarrollan su actividad en las mismas universidades $\mathrm{u}$ otros centros de investigación, mediante otros vínculos laborales y/o funcionariales ${ }^{24}$. En definitiva, los requisitos de titulación y el objeto del contrato no pueden permanecer al margen de la finalidad perseguida con el recurso a cualquier modalidad contractual y, en el caso del contrato SECTI, de estos elementos no se desprende un carácter formativo, sino de inserción laboral.

Este alejamiento del carácter formativo del contrato SECTI ya se percibía también en la STSJ del País Vasco de 2 de octubre de 2018 (núm. rec. 1670/2018), que ya planteaba dudas al respecto cuando indicaba que "aunque según el artículo 11.1 a) del ET el contrato en prácticas está dirigido a obtener una práctica profesional adecuada al nivel de estudios o de formación cursado, ello no quiere decir que la contratación de la actora sea equiparable al contrato en prácticas regulado en dicha norma (que como ya hemos indicado, es de aplicación supletoria para "lo no previsto") (...) No es equiparable porque, si acudimos al artículo 20 de la Ley 14/2011 en el que se regulan las distintas

\footnotetext{
${ }^{24}$ Esta concepción de los investigadores "Ramón y Cajal" como investigadores que no se encuentran en una fase de formación en sus carreras, sino que ya realizan actividades investigadoras ordinarias, ya se había puesto de manifiesto por algunos tribunales laborales en el anterior marco normativo -artículo 17.1.b) de la Ley 13/1986-. Vid. sobre esta cuestión, MORENO GENÉ, Josep: "La contratación "en prácticas" de los "investigadores Ramón y Cajal" según recientes sentencias del TSJ de Madrid", Aranzadi Social, núm. 1, 2008, p. 113 y ss. A tal efecto, la doctrina judicial elaborada en relación con el anterior contrato para la incorporación de investigadores al sistema de ciencia y tecnología establecía que siendo la esencia de todo contrato en prácticas la obtención de la práctica profesional adecuada al nivel de estudios previamente cursados por el trabajador, no debe acudirse a la vía de contratación de investigadores prevista en el artículo 17 de la Ley 13/1986 cuando la finalidad de la misma no sea que el investigador reciba una formación específica en los términos previstos en el artículo $11 \mathrm{ET}$, sino que, por el contrario, por poseer ya el mismo específicos y profundos conocimientos en su campo del conocimiento y, por tanto, encontrarse perfectamente capacitado para el desarrollo profesional de una labor de investigación, es contratado por el organismo de investigación para el desarrollo de las tareas propias y ordinarias del mismo.
} 
modalidades contractuales laborales del personal investigador, entre ellas se menciona el referido "contrato de acceso al sistema español de ciencia, tecnología e innovación" (...) de donde resulta que el último contrato de la actora se corresponde a una fase postdoctoral que pone fin a su etapa de formación como personal investigador (...) dando comienzo con el mismo a una situación distinta con un objetivo de búsqueda de perfeccionamiento y especialización profesional, que solamente es posible desarrollar con la nueva titulación de doctorado que se hizo constar en el nuevo contrato"25.

Por todo ello, resulta plenamente acertada la conclusión alcanzada por la STSJ del País Vasco de 22 de octubre de 2019 (núm. rec. 1699/2019) al considerar que "no se aprecia una diferencia en la actividad que no sea, en su caso, la que proviene del tiempo de ejercicio de la profesión, elemento que no sirve para diferenciar una actividad de otra. Tampoco los resultados, los proyectos o los fines se muestran desiguales".

En consecuencia, en el supuesto enjuiciado no se puede justificar una diferencia retributiva entre los investigadores contratados mediante el contrato SECTI y los investigadores contratados mediante la figura del personal investigador doctor contratado previsto en la normativa universitaria vasca, de modo que si la misma se produjera sería atentatoria al principio de igualdad, en tanto que dichas desigualdades retributivas implicarían una ruptura del principio de equivalencia retributiva entre trabajo y salario.

También resulta totalmente acertado que en aplicación del artículo 22.1.d) de la Ley de la Ciencia, el Tribunal condene a la Universidad a abonar las diferencias salariales existentes entre la retribución efectivamente percibida por los investigadores, fijada en sus respectivos contratos de trabajo, en base a las correspondientes ayudas a la investigación mediante las que se financiaban dichos contratos, y la retribución prevista para la figura del personal doctor investigador en el Decreto 41/2008, por considerar que nos encontramos ante un personal investigador que realiza actividades análogas.

A tal efecto, cabe distinguir entre los programas de ayudas a la investigación mediante los que se financian los contratos de los investigadores, en el supuesto enjuiciado, la convocatoria de ayudas "Ramón y Cajal", que se regirán por sus propias bases, de los contratos laborales que finalmente se formalicen a partir de dichas ayudas, que

\footnotetext{
${ }^{25}$ En otra parte de la sentencia, sin embargo, matiza esta primera afirmación al indicar que "ya nos hemos pronunciado en el sentido de que, por sus peculiares características, dicho contrato no es equiparable en cuanto a su finalidad al contrato de trabajo en prácticas regulado en el artículo 11.1 ET que resulta de aplicación supletoria, pero no es menos cierto que persiguiéndose con él la obtención de un elevado nivel de perfeccionamiento y especialización profesional, que indudablemente está por encima de la obtención de una práctica profesional adecuada a unos estudios recientes, no está exenta de una finalidad formativa, aunque de carácter más exclusivo".
} 
necesariamente deberán cumplir con lo establecido por la normativa laboral y, en particular, en el caso del contrato SECTI, con lo previsto en el artículo 22 de la Ley de la Ciencia. En otros términos, de ninguna manera puede permitirse que el mero hecho de que la contratación de estos investigadores doctores se financie a través de programas o convocatorias de ayudas a la investigación pueda justificar la pérdida o reducción de los derechos laborales básicos, incluidos los retributivos, correspondientes a este colectivo de investigadores. A este respecto, se ha puesto de manifiesto, sin bien, en relación a otro colectivo de investigadores también previsto en la Ley de la Ciencia, en particular, los investigadores predoctorales en formación, que dicho colectivo "son personas trabajadoras de los OPIs y de las universidades que no pueden ver cercenados los derechos laborales reconocidos al resto de personas trabajadoras de esas mismas entidades con la excusa de la financiación externa del contrato, so pena de incurrir en un supuesto de discriminación (artículo 14 CE)"26.

En esta dirección, resulta muy esclarecedora la STSJ del País Vasco (Sala de lo contencioso-administrativo) de 15 de mayo de 2019 (núm. rec. 1139/2017), si bien, también dictada en relación con los investigadores predoctorales en formación. En esta resolución judicial se conoce del recurso contencioso-administrativo interpuesto por una organización sindical contra una convocatoria de ayudas predoctorales, por considerar que la misma no se ajustaba, en materia salarial, a lo previsto en el artículo 21 de la Ley de la Ciencia, reguladora del contrato predoctoral.

En este punto, el TSJ (Sala de lo contencioso-administrativo) del País Vasco recuerda que estas convocatorias de ayudas a la formación de doctores se configuran como una actividad subvencional de la Administración. A partir de esta premisa, el Tribunal pone de manifiesto que en la convocatoria de ayudas se regula la "dotación" de dichas ayudas y se indica que la misma "se destinará al abono de la retribución bruta y de la cuota empresarial a la Seguridad Social del personal investigador por parte del centro empleador". Por el contrario, en el artículo 21 de la Ley de la Ciencia, por el que se regula el contrato predoctoral, se fija el mínimo retributivo de dicho contrato, no la cuantía de la ayuda a la investigación.

A partir de estas premisas, el Tribunal se plantea si la entidad convocante de las ayudas a la formación de doctores se encuentra obligada o no a fijar como cuantía de la ayuda la que resulte de aplicar el artículo 21 de la Ley de la Ciencia y, en respuesta a esta cuestión, concluye que "en todo caso (...) la Administración no viene obligada a cuantificar las ayudas en el $100 \%$ de los costes (retribución y cuota empresarial) que suponga la contratación de personal investigador con un contrato predoctoral'. Ahora bien, a

${ }^{26}$ Vid. BALLESTER LAGUNA, Fernando, "Claroscuros en la regulación del salario aplicable al contrato predoctoral”, Revista de Trabajo y Seguridad Social. CEF, núms. 449-450, 2020, p. 150. 
criterio del Tribunal, ello no impide que en el supuesto de que se formalice un contrato predoctoral financiado con estas ayudas, los trabajadores puedan acudir a la jurisdicción competente, que obviamente será la laboral, para cuestionar la retribución prevista en dicho contrato, si consideran que la que se les abona es inferior a la que establece el artículo 21 de la Ley de la Ciencia.

Extrapolando lo establecido en esta resolución judicial a la contratación de investigadores doctores a través del contrato SECTI, podemos distinguir entre la cuantía de la ayuda prevista en el correspondiente programa o convocatoria de ayudas a la investigación con las que se financia la contratación de los investigadores, en el supuesto enjuiciado, las ayudas "Ramón y Cajal", que se podrá fijar discrecionalmente por la entidad convocante de las mismas, de la retribución establecida para el contrato SECTI, que necesariamente deberá ajustarse a lo previsto en el artículo 22 de la Ley de la Ciencia, de modo que si la cuantía de las ayudas es inferior a la que corresponde a dicha modalidad contractual, la diferencia deberá ser complementada y, por tanto, sufragada, por la entidad contratante del investigador, a saber, la universidad u otro centro de investigación de adscripción y, en caso de no haberse actuado de este modo, el referido investigador doctor puede reclamar ante la jurisdicción social las diferencias salariales que correspondan, como así ha sucedido en el supuesto enjuiciado.

En este contexto, los problemas de gestión que se derivan para los gestores universitarios y de otros centros de investigación como consecuencia de los posibles desajustes existentes entre las convocatorias de ayudas a la investigación con las que se financia en la actualidad una parte importante de la contratación de los investigadores por parte de las universidades y otros centros de investigación y los contratos de trabajo que se formalizan en base a las mismas, únicamente se pueden salvar acudiendo a lo que se ha denominado como un "modelo mixto de cofinanciación del contrato", de modo que aquellos costes salariales que no sean asumidos por la entidad financiadora externa del contrato -el concreto programa o convocatoria de ayudas a la investigación-, deberán ser asumidos por la entidad contratante del investigador - universidad u otro centro de investigación- ${ }^{27}$.

${ }^{27}$ Vid. BALLESTAR LAGUNA, Fernando, “Claroscuros en la regulación...” cit. 


\section{Bibliografía.}

BALLESTER LAGUNA, Fernando, "Claroscuros en la regulación del salario aplicable al contrato predoctoral", Revista de Trabajo y Seguridad Social. CEF, núms. 449-450, 2020 .

GONZÁLEZ DÍAZ, Francisco Antonio, "El personal investigador en formación: una peculiar relación laboral", Revista del Ministerio de Empleo y Seguridad Social, núm. $118,2015$.

GONZÁLEZ DEL REY RODRÍGUEZ, Ignacio, “La contratación de investigadores por universidades públicas en la Ley de la Ciencia de 2011”, Revista de Trabajo y Seguridad Social. CEF, núm. 404, 2016.

LUJÁN ALCARAZ, José, "La contratación laboral del personal investigador ante la reforma laboral y la Ley de la Ciencia”, en AAVV.: Impacto de la nueva legislación en la educación superior y la investigación, Universidad de Sevilla, Sevilla, 2012.

MARINA JALVO, Belén, “Cuestiones esenciales del régimen jurídico del personal investigador al servicio de las universidades públicas tras la Ley 14/2011, de 1 de junio, de la Ciencia, la Tecnología y la Innovación", Revista General de Derecho Administrativo, núm. 30, 2012.

MOLINA HERMOSILLA, Olimpia, "Principales novedades en el ámbito de la gestión del personal docente e investigador. Un "pretendido modelo privilegiado" de acceso al empleo público", Revista de Trabajo y Seguridad Social. CEF, núm. 396, 2016.

MORENO GENÉ, Josep, "La actividad investigadora y la contratación laboral: una constante relación de desencuentros", Tirant lo Blanch, Valencia, 2005.

MORENO GENÉ, Josep, "El contrato de incorporación de investigadores al sistema de ciencia y tecnología”, Revista de Derecho Social, núm. 29. 2005.

MORENO GENÉ, Josep, "La contratación “en prácticas” de los “investigadores Ramón y Cajal” según recientes sentencias del TSJ de Madrid”, Aranzadi Social, núm. 1, 2008.

MORENO GENÉ, Josep, "La contratación laboral de los investigadores en la Ley de la Ciencia, la Tecnología y la innovación: ¿el final del estado de excepción laboral en la ciencia?", Revista de Trabajo y Seguridad Social. CEF, núm. 340, 2011. 
SANMARTÍN RODRÍGUEZ, Javier, "El carácter laboral del profesor universitario", Thomson Reuters Aranzadi Civitas, Madrid, 2012.

SERRANO ARGÜELLO, Noemí, "Modalidades específicas de contratación laboral del personal investigador. De la precarización al desideratum de su estabilidad en el empleo y consolidación profesional", Revista General del Derecho del Trabajo y de la Seguridad Social, núm. 36, 2014.

SIRVENT HERNÁNDEZ, Nancy, "Las modalidades de contratación laboral previstas en la LCTI específicamente para el personal investigador", en BALLESTER LAGUNA, FERNANDO (Coord.): El estatuto profesional del personal investigador contratado en régimen laboral, Innap Investiga, Madrid, 2016. 